\title{
Barrier Performance of CVD Graphene Films Using a Facile P3HT Thin Film Optical Transmission Test
}

\author{
Srinivasa Kartik Nemani and Hossein Sojoudi \\ Department of Mechanical, Industrial, and Manufacturing Engineering, University of Toledo, Toledo, Ohio 43606, USA \\ Correspondence should be addressed to Hossein Sojoudi; hossein.sojoudi@utoledo.edu
}

Received 25 October 2017; Revised 18 January 2018; Accepted 29 January 2018; Published 8 March 2018

Academic Editor: Ester Vazquez

Copyright (c) 2018 Srinivasa Kartik Nemani and Hossein Sojoudi. This is an open access article distributed under the Creative Commons Attribution License, which permits unrestricted use, distribution, and reproduction in any medium, provided the original work is properly cited.

\begin{abstract}
The barrier performance of CVD graphene films was determined using a poly(3-hexylthiophene) (P3HT) thin film optical transmission test. $\mathrm{P} 3 \mathrm{HT}$ is a semiconducting polymer that photo-oxidatively degrades upon exposure to oxygen and light. The polymer is stable under ambient conditions and indoor lighting, enabling P3HT films to be deposited and encapsulated in air. P3HT's stability under ambient conditions makes it desirable for an initial evaluation of barrier materials as a complimentary screening method in combination with conventional barrier tests. The P3HT test was used to demonstrate improved barrier performance for polymer substrates after addition of CVD graphene films. A layer-by-layer transfer method was utilized to enhance the barrier performance of monolayer graphene. Another set of absorption measurements were conducted to demonstrate the barrier performance of graphene and the degradation mechanism of graphene/P3HT over multiple wavelengths from 400 to $800 \mathrm{~nm}$. The absorption spectra for graphene/polymer composite were simulated by solving Fresnel equations. The simulation results were found to be in good agreement with the measured absorption spectra. The P3HT degradation results qualitatively indicate the potential of graphene films as a possible candidate for medium performance barriers.
\end{abstract}

\section{Introduction}

Graphene is considered for use in polymer barriers due to its structural impermeability [1]. Graphene's resolute hexagonal lattice structure of carbon lends itself to barrier because it has few defects and is impermeable to standard gases, including helium [2]. Such an inclusion can significantly hinder oxygen, gas, and water vapor transport, making it an intriguing prospect for incorporation into packaging composites. When incorporated appropriately, these atomically thin carbon sheets can significantly improve the physical properties of host polymers at extremely small loading. Exfoliated graphite was used to reinforce the thermoplastic polyurethane towards enhanced gas barrier performance [3]. Nitrogen permeation was remarkably reduced, demonstrating that exfoliated carbon sheets can be diffusion barriers in polymeric membranes. The oxygen transmission rate was decreased with increase in the volume percentage of graphene in graphene-based nanofiller [4]. Reduced graphene oxide (RGO) dispersed in polymers enables large scale barrier membranes with mechanical integrity [5]. The insulating properties of graphene oxide (GO) are desirable for barrier materials [6], but the hydrophilic nature of GO (compared with graphene) may not be suitable for organic electronic devices, for example, organic light-emitting diodes (OLEDs) and organic photovoltaics (OPVs), which degrade readily upon exposure to environmental oxygen and water vapor [7]. A sufficiently low free-volume for the graphene-based barrier material will result in both low oxygen transmission rates (OTRs) and low water vapor transmission rates (WVTRs). However, it is not clear if a minimized free volume can be obtained for a hydrophilic or hydrophobic graphenebased material. It might therefore be interesting to use a more hydrophobic insulating graphene-based material. The advancement in synthesis of high quality graphene using the chemical vapor deposition (CVD) technique may make it more viable for barrier applications as it is large scale and hydrophobic after being transferred to another substrate [8].

Major efforts have been made to develop high performance, optically transparent, and flexible encapsulation 
solutions to seal OPVs and OLEDs [9-13]. OTR and WVTR can be measured with the calcium thin film optical transmission test $[14,15]$. The calcium oxidative tests were used to determine the barrier performances of laminated graphene films [16], aluminum [17], and alumina [18]. This test measures the degradation of opaque $\mathrm{Ca}$ films, which transform into transparent and insulating $\mathrm{Ca}(\mathrm{OH})_{2}$ as the metal reacts with $\mathrm{O}_{2}$ and $\mathrm{H}_{2} \mathrm{O}$. The Ca test can be used to obtain WVTR values as low as $10^{-6} \mathrm{~g} / \mathrm{m}^{2} /$ day, which is the barrier requirement for OLEDs [12]. Ca has a very low Gibbs free energy of formation for its oxide [9], so the Ca film must be deposited under tightly controlled environmental conditions. The thermal evaporator also must be placed in a glove box filled with an inert gas to prevent degradation of $\mathrm{Ca}$ films during removal from the evaporator (following deposition), resulting in a high-cost test method with limited throughput. A modified electrical calcium test has widely been implemented for testing the barrier performances of materials in recent researches [17, 18]. However, the time constraints and the sensitivity of the tests to leakages, variation in temperatures, and calibration of the testing devices give room for distortion in results. Su et al. [19] used the glovebox technique to measure the water permeability by monitoring the weight loss in the liquid-filled container over time at normal temperature pressure. Direct gas permeation methods (e.g., the standardized $\mathrm{MOCON}^{\circledR}$ test [20-22] and mass spectrometry) [23] have limitations similar to those of the Ca test. These methods are nonetheless very important for barrier performance screening, as they are quantitative in nature and enable the determination of OTR and/or WVTR for barrier layers. However, a simple qualitative method for initial barrier screening would be useful, especially if it would complement the aforementioned quantitative test methods.

Poly(3-hexylthiophene) (P3HT) is an organic semiconductor widely used for organic field-effect transistors [24, 25] and in OPV devices [26]. P3HT degrades photo-oxidatively if exposed to $\mathrm{O}_{2}$ and light [26], resulting in an increase in optical transparency of the initially translucent P3HT films. Photolysis (film degradation due to light exposure in an inert atmosphere) of P3HT occurs several orders of magnitude slower than photo-oxidation [27]. No photolysis is expected to occur during the first 1000 hours of light exposure, providing a sufficiently long test period for the initial screening of barrier materials.

Herein, we performed an optical storage lifetime test qualitatively analogous to the Ca test, by exposing P3HT film covered with graphene to a light source, then taking light transmission measurements with a photodetector. P3HT films degrade very slowly in ambient conditions and typical indoor lighting; this allows for less stringent sample preparation for barrier testing. The barrier performance of large-area CVD-grown graphene was studied through transmission and absorption spectra measurements where the results were validated with the simulations. However, microscale cracks, voids, and vacancies may occur during the transfer process of CVD graphene films [28, 29]. Therefore, a layer-by-layer transfer of CVD graphene films was utilized to enhance the barrier performance. Although the degradation of $\mathrm{P} 3 \mathrm{HT}$ films occurs orders of magnitude slower than that of $\mathrm{Ca}$ films [30], the method can still be used for initial screening of barrier films in multilayer structures. The deposition techniques used in this study may be useful towards medium performance barrier systems, ranging from opaque food packaging to transparent device encapsulation, for example, for OLEDs and OPVs.

\section{Experimental}

2.1. Materials. P3HT was purchased from Sigma Aldrich (CAS number: 156074-98-5) and used without further processing. PMMA dissolved in $4 \%$ anisole and Cytop were purchased from MicroChem and AGC Chemicals, respectively. All films (P3HT, PMMA, and Cytop) are processed via spin-casting. Multiple samples of each film are made and characterized to ensure repeatability.

2.2. Preparation of Graphene-PMMA Substrate. Multilayer graphene films were grown by chemical vapor deposition (CVD) on a $25 \mu \mathrm{m}$ thick $\mathrm{Cu}$ foil at $1000^{\circ} \mathrm{C}[8,31-33]$, and a layer of PMMA as an auxiliary supportive material was spun-cast and cured on the as-grown graphene. $\mathrm{Cu}$ foil was then acid-etched away in $\mathrm{FeCl}_{3}$ solution overnight, and the PMMA/graphene sample was washed in deionized water several times in preparation for transfer onto the target substrate. Finally, the auxiliary PMMA was removed in an acetone bath.

\subsection{Transfer of Mono and Multilayer Graphene. With PET} as our substrate, we spun-cast poly(methyl methacrylate) (PMMA, purchased from MicroChem) dissolved in anisole ( $4 \%$ volume) before transferring the graphene film on top of the PET/PMMA bilayer.

To complete the polymer "sandwich," a layer of Cytop (AGC Chemicals) was spun-cast on top of the graphene film to serve as both a protective and an insulating layer. The polymer barrier was made from a spin-coated commercial Cytop ${ }^{\mathrm{TM}}$ perfluorinated polymer barrier deposited from solution [34].

A proprietary seal was used to encapsulate the PET/ multilayer film over the top of the P3HT sample. The gap between the P3HT film and the multilayer was filled with air, since the entire encapsulation process was performed under ambient conditions, in order to demonstrate the simplicity of the method.

2.4. Characterization of the Films. A J. A. Woollam variable angle spectroscopic ellipsometer was used to measure the thickness of spun-cast films (P3HT: 100 nm; Cytop: $110 \mathrm{~nm}$; PMMA: $\sim 150 \mathrm{~nm}$ ) ensuring uniformity of the films/ processes. A HORIBA Scientific Raman spectrometer with an excitation wavelength of $532 \mathrm{~nm}$ laser light was used to verify presence of monolayer and multilayer graphene films. Laser power was kept below $0.5 \mathrm{~mW}$ to avoid laser-induced heating. A 50x objective lens was used to focus the laser on the graphene samples during the Raman measurements. A compact UV-Vis $2600 / 2700$ spectrometer was used to measure transmittance of graphene films. An Ocean Optic HR4000Cg-UV-NIR spectrometer was used to measure 
the absorption spectra of bare P3HT, P3HT/PMMA, and $\mathrm{P} 3 \mathrm{HT} / \mathrm{monolayer}$ graphene/PMMA samples.

\section{Results and Discussion}

The viability of the P3HT test was investigated by preparing spin-coated P3HT films with and without an encapsulating polymer barrier layer (Cytop). Figure 1(a) shows schematics of sample architectures. The samples were exposed to a $22 \mathrm{~W}$ fluorescent lamp and their transmission was frequently recorded using a photodetector until no further change in visible light transmission is observed. For each set of experiments multiple bare $\mathrm{P} 3 \mathrm{HT}$ samples are tested as control samples; once no further change in visible light transmission is recorded for a sample it is assumed that the P3HT film on that sample is fully degraded. Figure 1(b) shows P3HT degradation (change in visible light transmission of $\mathrm{P} 3 \mathrm{HT}$ film) as a function of time under ambient conditions, for P3HT films with and without a Cytop encapsulating layer. In the beginning of the tests, samples experience no exposure to the fluorescent lamp and therefore no change in P3HT transmission (degradation of $0 \%$ ) is present. $\mathrm{P} 3 \mathrm{HT}$ degradation of $100 \%$ refers to fully degraded P3HT film in a given sample, when no further change in P3HT transmission is observed (both for bare P3HT and multilayer samples containing a P3HT film). The degradation curves looked almost identical between duplicates of each sample (not shown), suggesting that any nonuniformity in intensity from the light source is negligible. Test samples could also be exposed to light along with elevated temperature and humidity to provide typical accelerated testing conditions. Higher temperatures increase the diffusion rates through the barriers and the degradation rates of the underlying $\mathrm{P} 3 \mathrm{HT}$ films.

If the P3HT thin film optical transmission test has the potential to be used as a qualitative, comparative initial screening method of barrier performance, then the barriers with one or more additional layer(s) should provide measurable increases in $\mathrm{P} 3 \mathrm{HT}$ lifetime compared with barriers having no additional layer(s). To further investigate the consistency in the P3HT test method, the P3HT lifetimes of polymer substrates were measured before and after addition of multilayer graphene. Graphene's barrier properties were evaluated through the fabrication of polymer multi-layered structures with and without graphene, schematically shown in Figure 1(a)(i-ii) as bare P3HT and P3HT/Cytop and (iii-iv) as P3HT/PMMA/Cytop and P3HT/Multilayer graphene/Cytop, respectively. Figure 1(e) shows a photo of a multi-layer graphene transferred onto a fully transparent PET substrate. UV-Vis spectroscopy measurements were performed on the multilayer graphene samples which exhibited around $84 \%$ light transmission at $550 \mathrm{~nm}$ wavelength (Figure 1(e)). Raman spectroscopy measurement was also performed on the multilayer graphene sample transferred on a Si-substrate, indicating presence of graphitic peaks ( $G$ and $2 \mathrm{D}$ bands) and lack of any defect (D) band (Figure 1(d)). As shown in Figure 1 (c), trapped $\mathrm{O}_{2}$ resulted in $\sim 10 \%$ P3HT degradation during the first 62 hours of light exposure for the polymer structure with multilayer graphene (P3HT/multilayer graphene/Cytop), while the structure without graphene and instead with a layer of PMMA (P3HT/PMMA/Cytop) had degraded 2.5 times more during the first 62 hours of light exposure. It has been shown that the photo-induced charge transfer phenomena of graphene in contact with $\mathrm{P} 3 \mathrm{HT}$ can be neglected due to a limited lifetime of the separated carriers and quenching effect of $\mathrm{P} 3 \mathrm{HT}$ in the presence of carbon based materials such as SWCNTs [35]. It has also been shown that the light absorption of graphene films between di-electric layers can be enhanced [36,37], resulting in a significant delay in build-up of steady-state regime in the light source. Therefore, reduced power of light initially incident onto the P3HT film can lead to initially slower P3HT degradation in structures with the graphene film. The full P3HT degradation occurred after $~ 500$ hours of fluorescent light exposure in structures with PMMA, whereas it took $\sim 680$ hours for P3HT to fully degrade in structures with multilayer graphene. Graphene is composed of closely packed carbon atoms in a two-dimensional structure, giving rise to its barrier functionality. Therefore, the addition of relatively thin multilayer graphene (between 10 and $20 \mathrm{~nm}$ thick) resulted in an approximately 25\% longer P3HT lifetime, when replaced with a relatively thick PMMA layer. However, after initially delayed P3HT degradation in structures with graphene when compared to structures with PMMA, both structures experienced close P3HT degradation rate. While no considerable voids were observed in graphene layers, micro size cracks can occur during the transfer process of graphene. Therefore, less significant improvement in barrier functionality observed in the apparent steady-state rate might be attributed to defects, which occur during the transfer of the multilayer graphene from the copper substrate. Accordingly, a new strategy utilizing monolayer graphene will be employed to qualitatively examine $\mathrm{P} 3 \mathrm{HT}$ as a barrier-screening thin film.

To this end, barrier performance of a monolayer graphene is tested using new structures without a proprietary seal and encapsulating Cytop as shown in Figure 2. Monolayer graphene was synthesized thorough a similar method explained earlier except with different precursor gases and under vacuum pressure. Raman spectroscopy was performed on transferred graphene samples and indicated presence of graphitic bands ( $\mathrm{G}$ and 2D bands) and lack of defect band (D band). The $2 \mathrm{D}$ to $\mathrm{G}$ band intensity ratios, $I_{2 \mathrm{D}} / I_{\mathrm{G}}$, of the samples synthesized and transferred were higher than 2.3, and the full width at half maximum (FWHM) of the 2D band was around $30 \mathrm{~cm}^{-1}$, which is indicative of monolayer CVD graphene (Figure 2(c)). Figure 2(d) shows UV-Vis spectroscopy performed on graphene samples with around $97 \%$ transparency measured at $550 \mathrm{~nm}$ wavelength, which is another indicative of a single-layer graphene [38]. Supportive PMMA used in the transfer process was not removed to minimize the defects in the barrier film in order to obtain better performance. Figure 2(a) shows different structures tested to verify this improvement. The same thickness of PMMA was used or deposited for the graphene transfer or spin-casting process, respectively. As expected, the structures with PMMA films show a generally higher degradation time for P3HT. Figure 2(b) shows the P3HT degradation in various structures, indicating barrier performance of a thin monolayer graphene 
(iii)

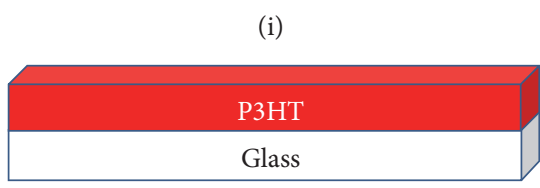

(ii)

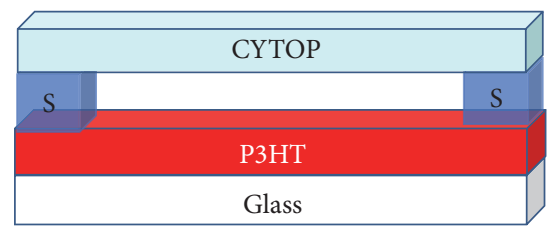

(a)

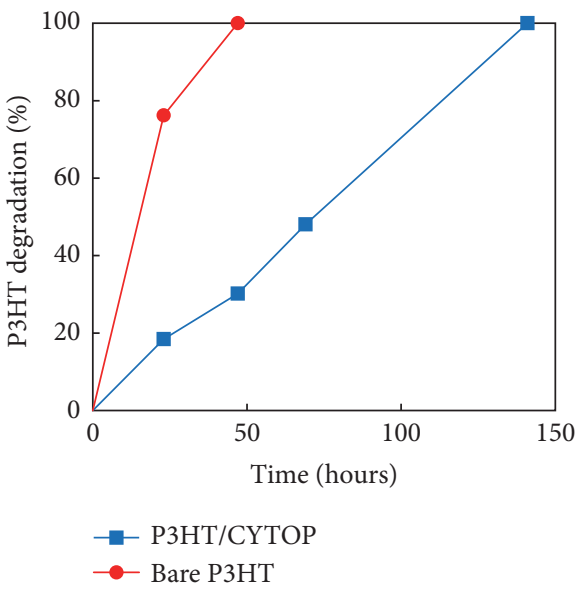

(b)

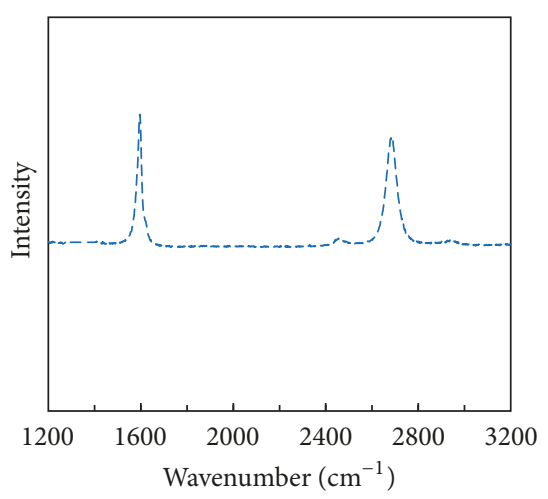

(d)

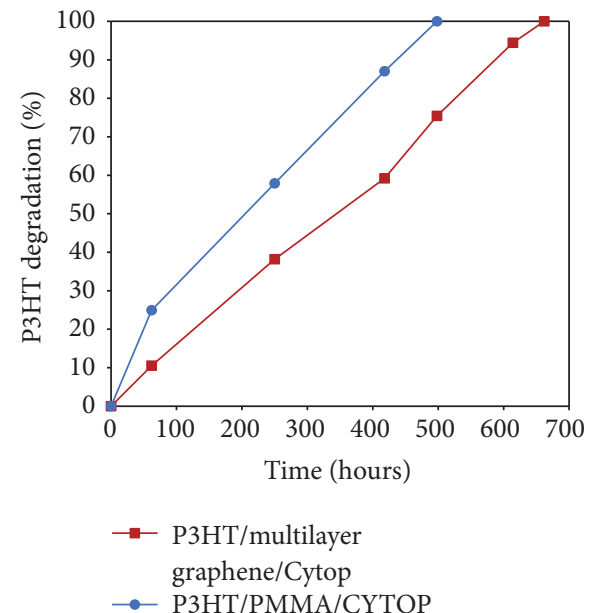

(c)

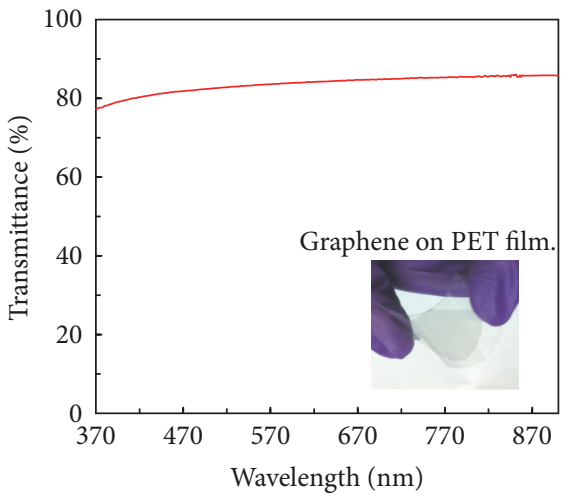

(e)

FIgURE 1: (a) Sample architectures for P3HT film (i), P3HT encapsulated with Cytop ${ }^{\mathrm{TM}}$ (ii), P3HT/PMMA (spin-cast) encapsulated with Cytop (iii), and P3HT with transferred multilayer graphene encapsulated with Cytop (iv). All structures were prepared using a proprietary seal. (b) Degradation rate of the P3HT film as a function of time with encapsulating Cytop (P3HT/Cytop, squares) and without encapsulating Cytop (Bare P3HT, circles). P3HT degradation of $100 \%$ refers to fully degraded P3HT film in a given sample, when no further change in P3HT transmission is observed (both for bare P3HT and P3HT/Cytop). Bare P3HT film as a control sample is used in each set of measurements during photodegradation under the fluorescent lamp. (c) P3HT degradation as a function of time for P3HT with spun-cast PMMA and encapsulated with Cytop (P3HT/PMMA/Cytop, circles) and P3HT with transferred multilayer graphene encapsulated with Cytop (P3HT/multilayer graphene/Cytop, squares). The tests and sample storage were done under ambient conditions ensuring uniformity in the intensity of the light source. (d) Raman spectra of multilayer graphene transferred on a Si-substrate which indicates presence of $\mathrm{G}$ and $2 \mathrm{D}$ bands and lack of any defect (D) band. The $I_{2 \mathrm{D}} / I_{\mathrm{G}}$ ratio is $\sim 0.83$; full width at half maximum (FWHM) of the $2 \mathrm{D}$ band is $\sim 55 \mathrm{~cm}^{-1}$ indicative of multilayer graphene. (e) UV-Vis spectroscopy of the multilayer graphene shows approximately $84 \%$ transmittance at $550 \mathrm{~nm}$ wavelength, suggesting presence of multilayer graphene film. Multilayer graphene film transferred to a PET substrate (insert). 


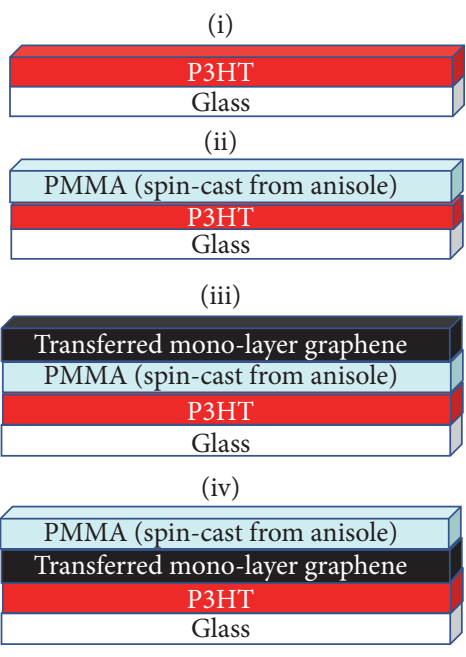

(a)

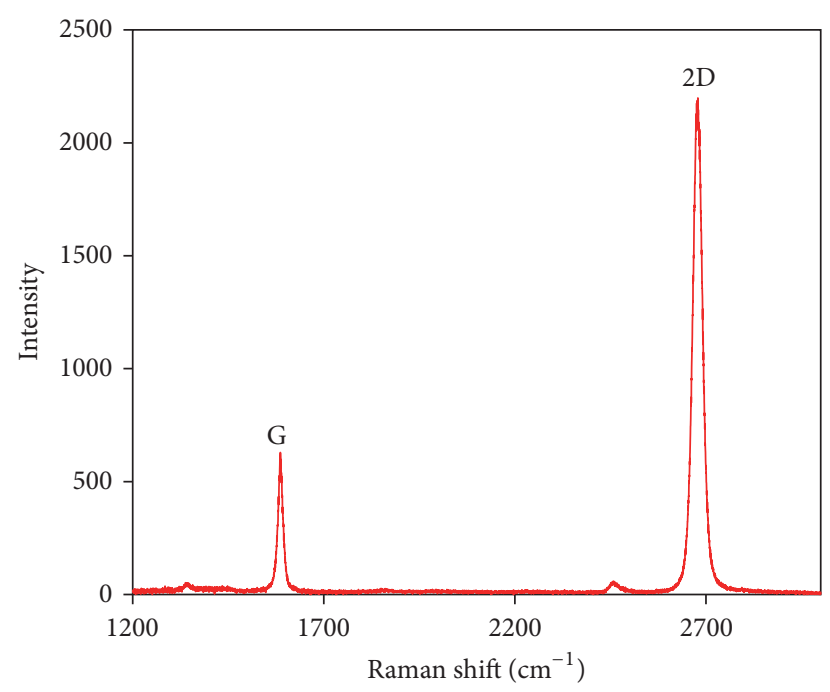

(c)

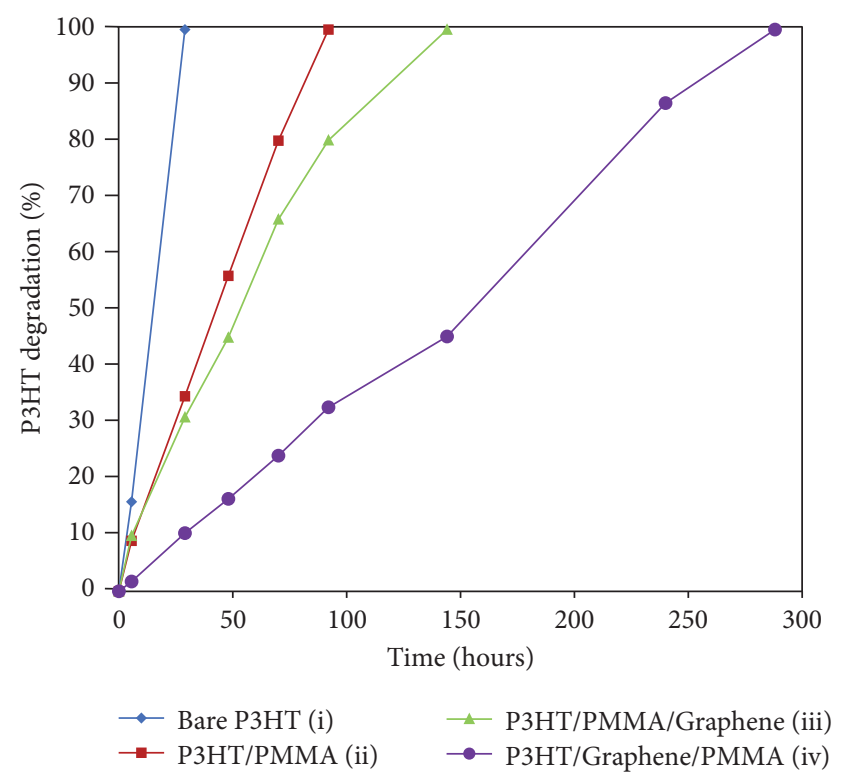

(b)

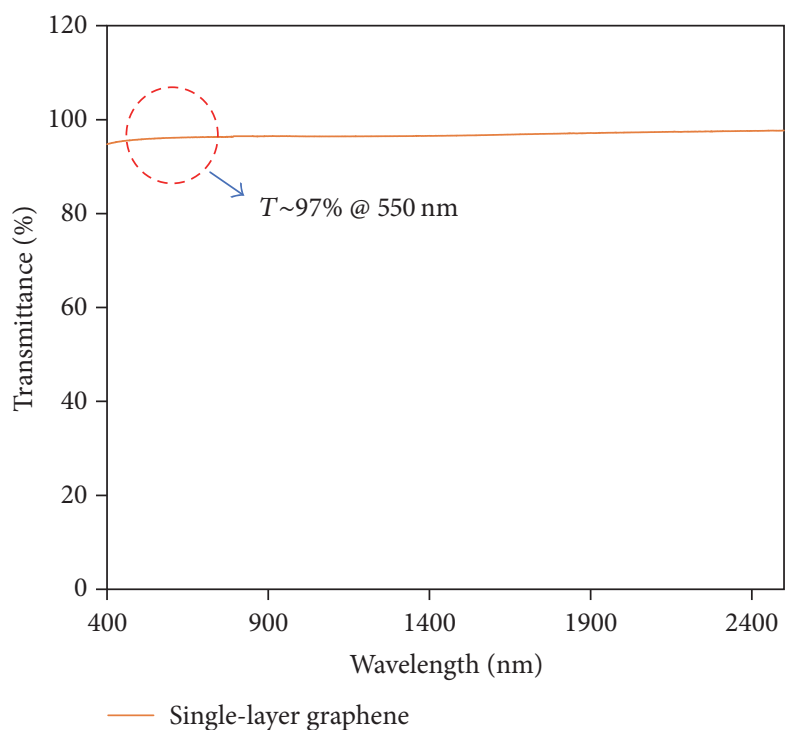

(d)

FIgure 2: (a) Sample architectures for P3HT films spun-cast on glass without any proprietary seal. (i) Bare P3HT, (ii) with PMMA spun-cast from anisole, (iii) P3HT with PMMA (spun-cast) and monolayer graphene (supportive PMMA removed), and (iv) P3HT with monolayer graphene and PMMA (transferred with graphene). (b) P3HT degradation as a function of time for structures i-iv. The removal of PMMA from monolayer graphene during its transfer generates cracks in the film, which results in depleted barrier performance of monolayer graphene, when comparing PMMA/graphene (structure iii) with graphene/PMMA (structure iv). Twofold reduction in P3HT degradation rate is attributed to minimized cracks in graphene film, which can be typically induced by removing the supportive PMMA layer. (c) Raman spectroscopy of graphene with $I_{2 \mathrm{D}} / I_{\mathrm{G}}=2.3$ and full width at half maximum (FWHM) of the $2 \mathrm{D}$ band around $30 \mathrm{~cm}^{-1}$, indicative of monolayer CVD graphene. (d) shows UV-Vis spectroscopy performed on graphene samples with around $97 \%$ transparency measured at $550 \mathrm{~nm}$ wavelength, which is another indicative of a monolayer graphene.

when comparing structures (iii) and (iv) with (i) and (ii). It is to be noted that the slight enhancement in degradation of the bare P3HT film in Figure 2(a) when compared to Figure 1(a) is due to elimination of the proprietary seal in the new sample architectures. It is observed that the P3HT degradation time is doubled for structure (iv) where PMMA is transferred with graphene, compared to structure (iii) where it is spuncast. This indicates the contribution of defects during transfer process of graphene towards its reduced barrier performance. On the other hand, an addition of the monolayer graphene composed of closely packed carbon atoms without any cracks resulted in an approximately threefold longer P3HT lifetime 
(i)

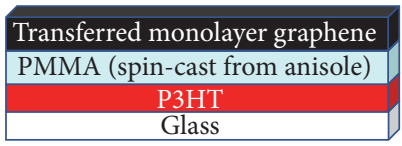

(ii)

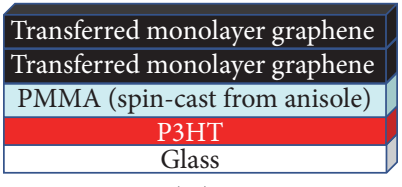

(iii)

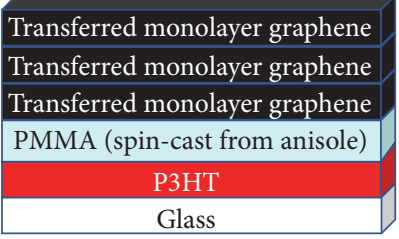

(a)

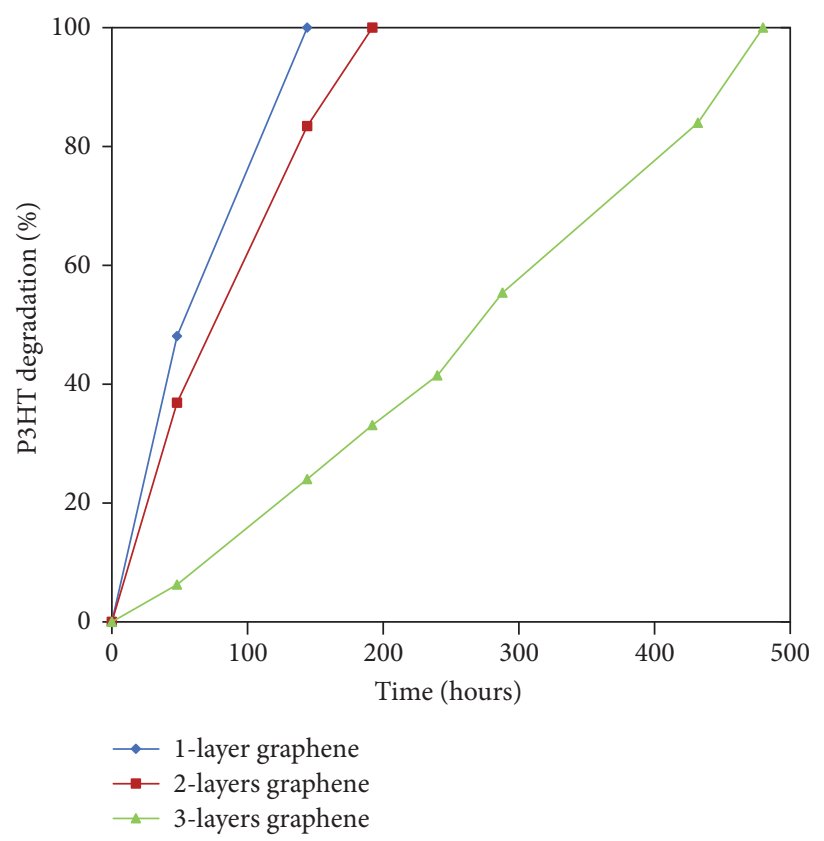

(b)

FIGURE 3: (a) Schematic of a layer-by-layer transfer of monolayer graphene for enhanced barrier performance (i) P3HT/PMMA (spuncast)/monolayer graphene, (ii) P3HT/PMMA (spun-cast)/monolayer graphene (2 layers), (iii) P3HT/PMMA (spun-cast)/monolayer graphene (3 layers), (b) P3HT degradation as a function of time indicating enhanced barrier performance by adding more layers of monolayer graphene which covers possible cracks in the monolayer graphene resulting in lower slope for the 3-layer graphene structure.

(see Figure 2(b); compare results for structures (ii) and (iv)). It must be noted that removing PMMA during graphene transfer process can introduce micron size cracks in the film, negatively influencing its barrier performance. This can be seen from the twofold decrease in the slope of P3HT degradation curve, when comparing structures (iii) and (iv) in Figure 2(a) or from approximately similar P3HT degradation rate, observed in samples of structures (ii) and (iii).
A layer-by-layer transfer of monolayer graphene was utilized to mask the cracks in the monolayer graphene. Figure 3(a) shows different structures tested using different numbers of monolayer graphene as barrier. Structures in Figure 3(a) (i, ii, and iii) contain identical layers of spuncast and cured PMMA. Therefore, the longer full P3HT degradation time and decrease in $\mathrm{P} 3 \mathrm{HT}$ degradation rate in structure (ii) compared to structure (i) are due to the presence of another monolayer of graphene, with 30\% longer P3HT 
lifetime. Addition of another monolayer graphene as shown in structure (iii) results in almost three times longer degradation for the P3HT film. This indicates a significantly enhanced barrier performance through the layer-by-layer approach, without using a seal and/or encapsulation with Cytop, where possible cracks and wrinkles in the monolayer graphene were covered by addition of two monolayer graphene films. Presence of a monolayer graphene without any cracks results in almost threefold enhancement in P3HT lifetime (Figure 2, compare results for structures ii and iv). However, presence of potential cracks due to PMMA removal negatively influences barrier performance of monolayer graphene. Nevertheless, three layers of monolayer graphene obtained from the layerby-layer transfer method enable covering of potential cracks in each layer and result in improved barrier functionality of stacked layers. This barrier functionality is not observed in a graphene film that is synthesized as a multilayer and then transferred in a single step (see results of Figure 1).

To further understand both the degradation process of P3HT coated with a thin layer PMMA and graphene and the barrier performance of graphene, the absorption spectra of bare P3HT, P3HT/PMMA, and P3HT/monolayer graphene/ PMMA were measured. An Ocean Optic HR4000Cg-UVNIR spectrometer was used for this purpose. It must be noted that the measured values are in fact absorbance $(A)$ and reflectance $(R)$ which is 1-transmittance (1-T). Samples were prepared with the same method explained earlier to ensure consistency. The structures of the samples are as summarized in Figure 2(a). Figure 4(a) shows the UV-Vis absorption spectra measured for bare P3HT after being exposed to light. Two peaks at $525 \mathrm{~nm}$ and $550 \mathrm{~nm}$ and one shoulder at $600 \mathrm{~nm}$ can be observed. These three bands can be attributed to the $\pi-\pi^{*}$ transition. The shoulder comes from highly ordered domains $[26,27,39]$. During photodegradation, a decay in the intensity of the peaks accompanied by a shift to lower wavelengths can be observed. In addition, a change in the interchain order occurs, which results in a disappearance of the shoulder. The former change is indicative of a reduction in the conjugation length of the macromolecular backbone; this leads to photo bleaching of the polymer, as no peak was observed after 128-hour exposure to direct light [26].

The interaction between $\mathrm{P} 3 \mathrm{HT}$ and other polymers during photo-oxidation has previously been investigated [39]. The absorption spectra of thin films obtained by spincoating a blend of P3HT:PCBM from solution showed a significant blue shift. This change is attributed to both the destruction of ordering of P3HT chains and a non-photoinduced charge transfer between P3HT and PCBM [40, 41]. $\mathrm{Wu}$ et al. developed a high-resolution photoluminescent electron beam resist using polymer blends consisting of P3HT and PMMA, where they studied the optical properties by incorporating different polymer ratios [41]. However, the authors are not aware of any study of absorption spectra in PMMA/P3HT bilayers. In the present work, we investigated the effect of a graphene layer on the absorption spectra of bilayer P3HT/PMMA to correlate performance of the barrier layer(s) with any changes in the absorption properties of P3HT. Figure 4(b) shows the absorption spectra for bilayer
P3HT/PMMA. The photo-oxidation process was considerably slowed down when a PMMA layer was spin-coated on P3HT, resulting in full degradation of P3HT after approximately 300 hours (not shown). The comparison with the behavior of bare $\mathrm{P} 3 \mathrm{HT}$ demonstrates that the polymer degradation rate is slower with a spin-cast PMMA overcoat barrier, resulting in around three times longer P3HT lifetime. The wave patterns observed in the absorption spectra of $\mathrm{P} 3 \mathrm{HT} /$ PMMA are believed to be due to an interface effect between the P3HT and PMMA layers and will be discussed in further details later in this paper. Figure 4(c) shows the absorption spectra for P3HT/graphene/PMMA. The wave patterns were not observed in this sample, which comprised a bilayer graphene/PMMA transferred on P3HT without allowing a direct contact between P3HT and PMMA. Conjugated polymers like P3HT show anisotropic light scattering due to the presence of crystalline and amorphous domains in thin films [42] which effect their net absorption in sandwiched structures, causing variable absorption of graphene plus $\mathrm{P} 3 \mathrm{HT}$ bilayer films as seen in Figure 4(c). Comparison of the spectra for different structures confirm the expected behavior that the sample covered with a monolayer of graphene has higher absorption (see Figure 4(d)). The change in the absorption spectra after being exposed to direct light for 128 hours was less pronounced for P3HT/graphene/PMMA. The observed blue shift in P3HT spectra after degradation was smaller and the shoulder did not disappear after 128 hours. This indicates the presence of $\mathrm{P} 3 \mathrm{HT}$ as well as the effect of the barrier performance of PMMA and PMMA/graphene. When the P3HT degradation is low, the absorption of the films exhibits a small blue shift to $515 \mathrm{~nm}$. As the P3HT degrades more, the absorption spectra show a significant blue shift. Measurements were repeated up until P3HT was fully degraded, which occurred after almost 600 hours. The comparison of the measurements obtained from Ocean Optic with transmittance results gives more insight into performance of the graphene film. While for the same structure (Figure 2(d)) transmittance measurements indicate fully degraded $\mathrm{P} 3 \mathrm{HT}$, absorption data are not indicative of fully degraded P3HT. It is to be noted that the transmittance values are relative while the absorption values are absolute. While a 100\% degraded P3HT sample was assumed completely transparent with respect to its initial state, the absorption values are not relative. Differences in light sources and detection mechanisms of two methods have to be noted as well. Transmittance values are obtained using a simple in-house photodetector while absorption Ocean Optic measurements are conducted in a black box with an InGaAs photodiode for detection and a variable blazed grating of 50 micron slit size and optical resolution of 1.5 microns (range $250-1050 \mathrm{~nm}$ ). Therefore, the transmittance values are used to determine the rate of $\mathrm{P} 3 \mathrm{HT}$ degradation (\%) qualitatively, while the absorbance values are used to understand the photodegradation mechanism of the P3HT film.

The wave patterns in the absorption spectra of P3HT/ PMMA may be explained by a lower reflection of light from the PMMA film, which can be partially attributed to a difference in the refractive index between PMMA and P3HT. To this end, the refractive indexes for P3HT, PMMA, 

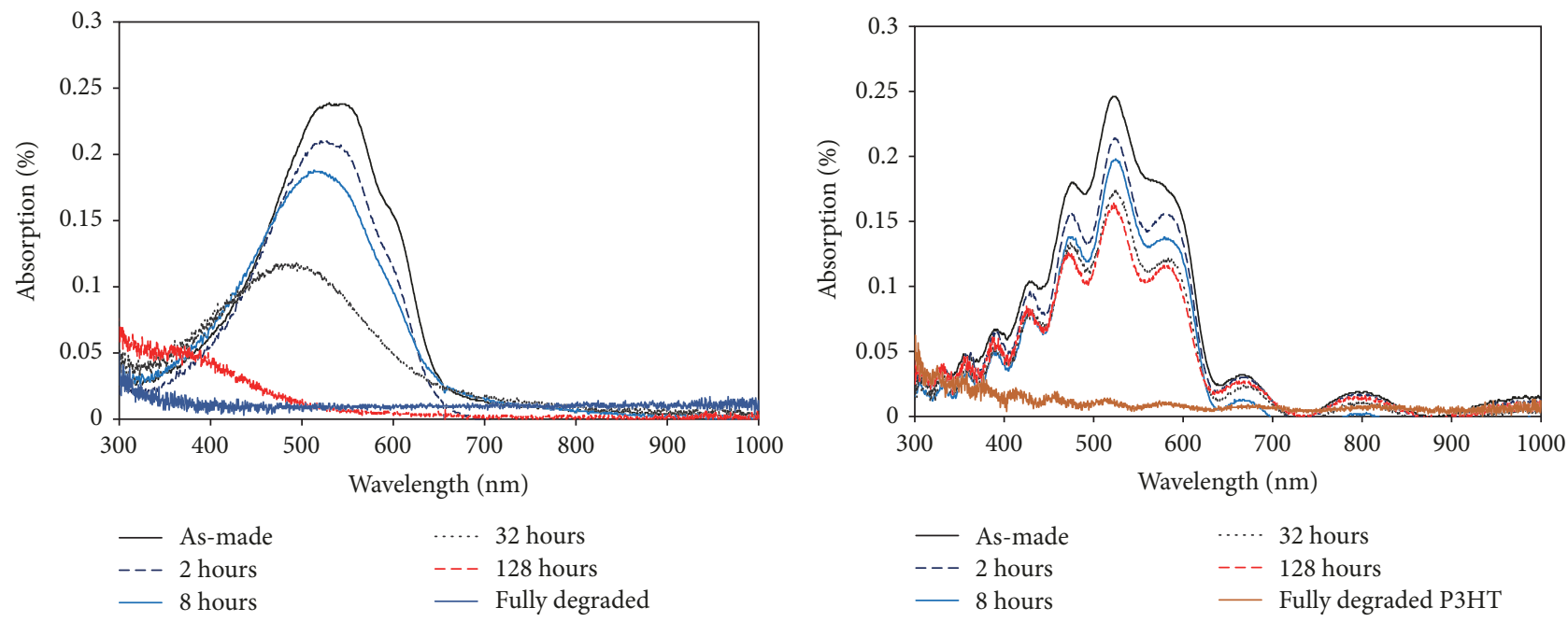

(a)
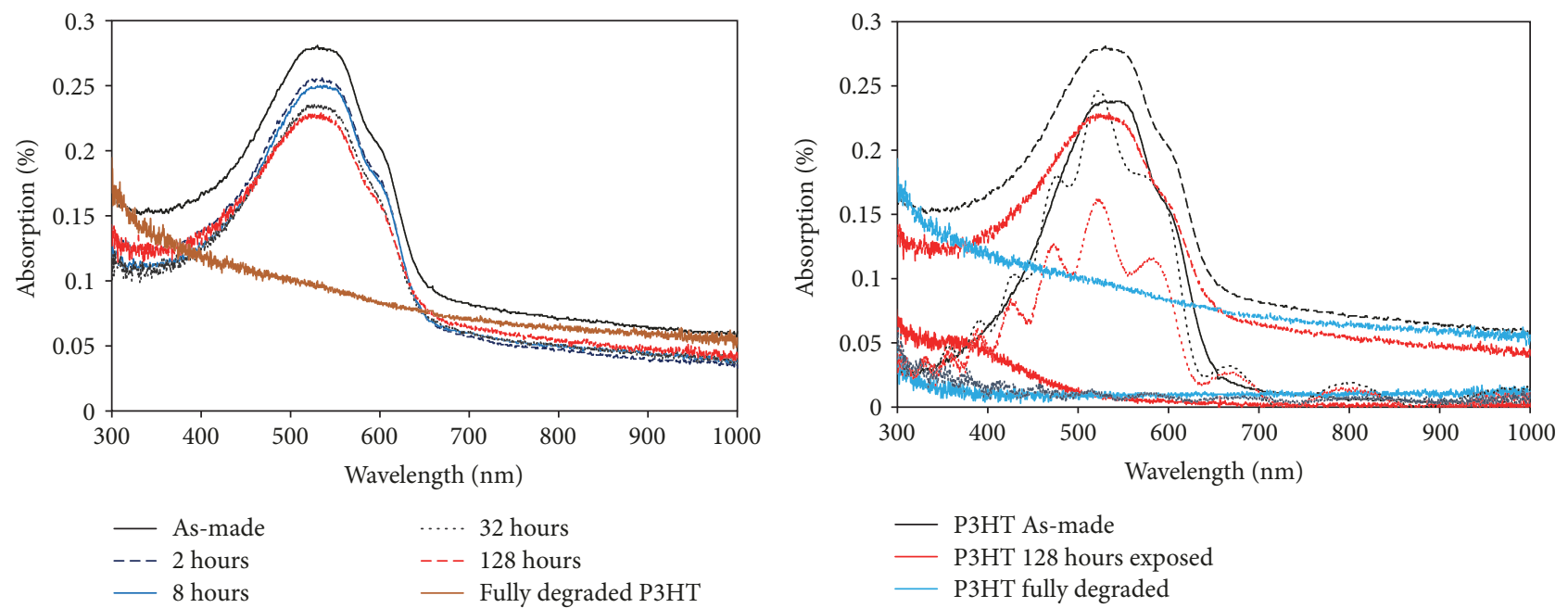

(c)

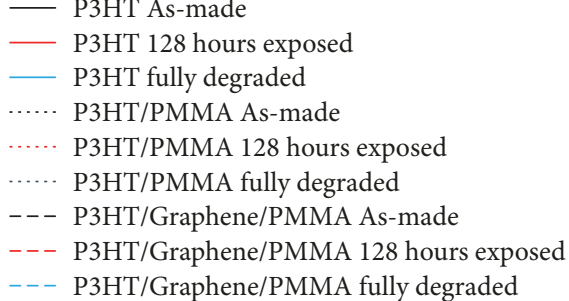

(d)

Figure 4: Absorption spectra measured at different time interval. (a) Bare P3HT, (b) P3HT/PMMA (spun-cast), (c) P3HT/graphene/PMMA (PMMA transferred with graphene). The photooxidation process slowed after addition of a PMMA layer (b), resulting in complete degradation after 300 hours of exposure, 3 times the life of bare P3HT. Wave patterns are not observed in (c) as the P3HT and PMMA were separated and not in direct contact. (d) Comparison of absorption spectra of structures in (a), (b), and (c) (as-made, after 128 hours of exposure to light, and fully degraded at $\sim 300$ hours). It is observed that the sample covered with monolayer graphene has higher absorption in comparison to other tested structures. The schematic of the samples tested is shown in Figure 2.

and graphene were measured and the absorption curve was calculated by solving Fresnel equations. The film thickness and refractive index $(N=n+i k)$ were obtained from recently published data [43]. These data were used to obtain absorption through solving Fresnel equations. Figure 5 shows the simulation results and its comparison with the measurements. It is observed that the simulation results for bare $\mathrm{P} 3 \mathrm{HT}$ and bilayer P3HT/PMMA were in good agreement with the experimental measurements (see Figures 5(a) and 5(b)). The disappearance of the wave patterns in absorption spectra of
P3HT/graphene/PMMA (Figure 5(c)) is due to a different roughness of the PMMA film in this structure. As explained earlier, PMMA was transferred with graphene that has been spin-coated on copper foil, which has a rougher substrate. This effect has not been considered in the simulations.

As already mentioned, photolysis (film degradation due to light exposure in an inert atmosphere) of P3HT occurs at a rate which is several orders of magnitude slower than photooxidation [26]. No significant change in P3HT absorbance can be expected within 1000 hours of light exposure (in an 


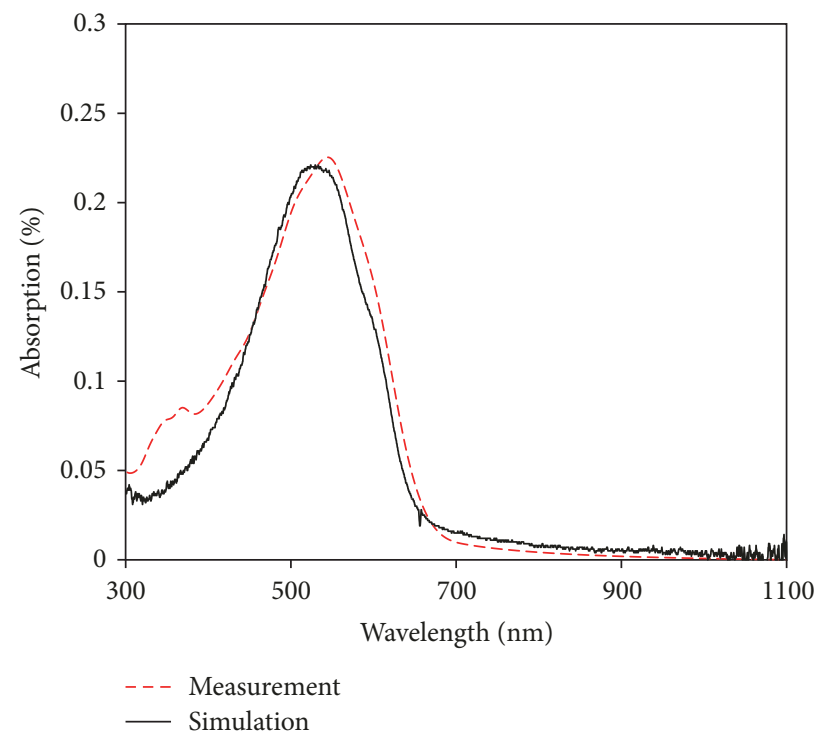

(a)

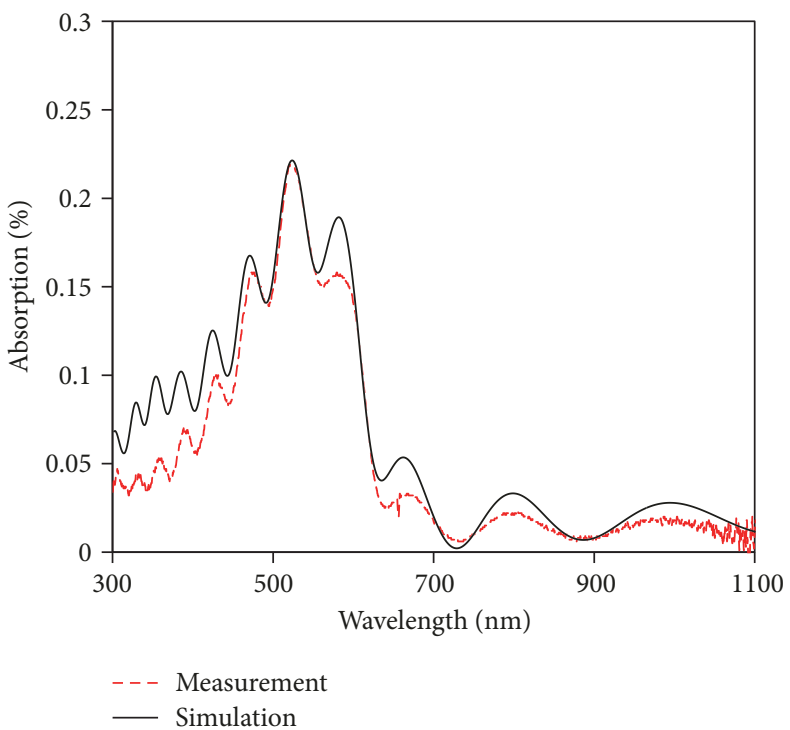

(b)

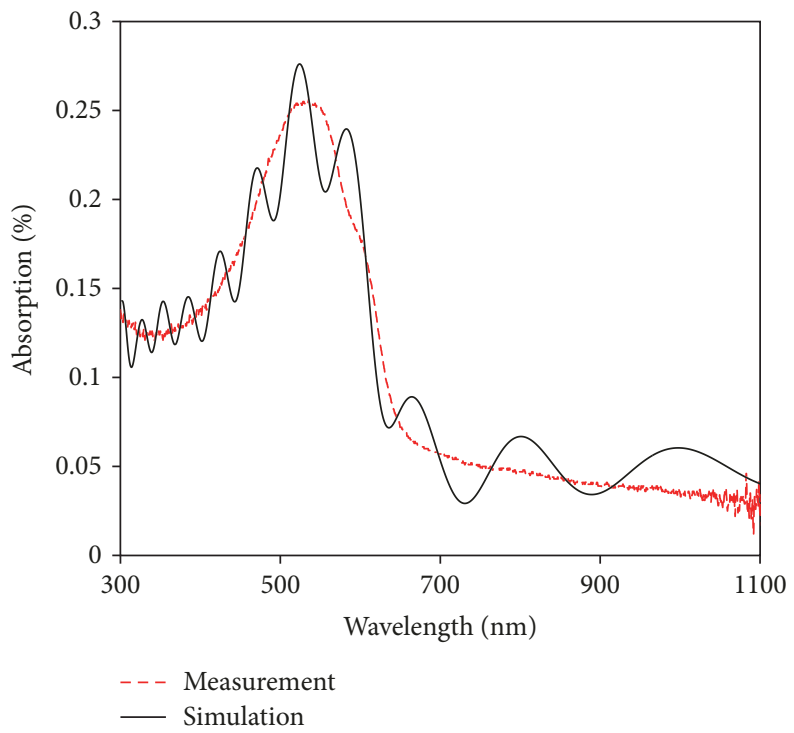

(c)

Figure 5: Absorption spectra measurements versus simulation results for (a) bare P3HT, (b) P3HT/PMMA (spin-cast), and (c) P3HT/graphene/PMMA (PMMA transferred with graphene). The plots for bare P3HT and P3HT/PMMA are in good agreement with the simulation results. It is observed that the wave patterns disappear in the absorption spectra of (c) P3HT/graphene/PMMA, due to the variable roughness of the PMMA layer. The simulations were performed assuming negligible roughness factor.

inert atmosphere), so photolysis did not contribute to P3HT degradation in this study. Low permeability and light resistant barriers, with a barrier performance that is not reduced due to photooxidative reactions in air, should exhibit negligible P3HT degradation after 1000 hours of light exposure for possible use in commercial OLED and/or OPV applications.

\section{Conclusions}

In summary, we have demonstrated a P3HT thin film optical transmission test which can be used to qualitatively screen the barrier performance of thin films. The deposition of
P3HT films can be done in air unlike the analogous calcium thin film optical transmission test, allowing a simplified fabrication process for the P3HT test. The reproducibility of the P3HT test was confirmed by comparing unencapsulated P3HT films with those having been encapsulated with Cytop. The P3HT test was used to screen the barrier performance of multilayers and monolayer graphene, which indicates that it was still sensitive enough to discern the barrier performance of a monolayer of carbon atoms. The influence of defects introduced in the transfer process of CVD graphene was shown to be critical for the tested structures with and without removing supportive PMMA. 
Another set of layer-by-layer polymer/graphene structures was made towards better barrier performance, showing almost three times longer P3HT lifetime with the addition of three monolayers of graphene. Absorption measurements revealed wave patterns for $\mathrm{P} 3 \mathrm{HT} / \mathrm{PMMA}$ bilayers, indicating an interface effect for P3HT/PMMA which was not found for $\mathrm{P} 3 \mathrm{HT} /$ graphene/PMMA. Simulation results confirmed the wave patterns obtained in the absorption measurements for $\mathrm{P} 3 \mathrm{HT} / \mathrm{PMMA}$. The $\mathrm{P} 3 \mathrm{HT}$ may be used as a simple way to screen the improvements of simple barrier structures prior to more detailed $\mathrm{Ca}$ or other permeation rate tests, which are costly and take more time for sample preparation.

\section{Conflicts of Interest}

The authors declare no competing financial interests.

\section{References}

[1] J. S. Bunch, S. S. Verbridge, J. S. Alden et al., "Impermeable atomic membranes from graphene sheets," Nano Letters, vol. 8, no. 8 , pp. $2458-2462,2008$.

[2] O. Leenaerts, B. Partoens, and F. M. Peeters, "Graphene: A perfect nanoballoon," Applied Physics Letters, vol. 93, no. 19, Article ID 193107, 2008.

[3] H. Kim, Y. Miura, and C. W. Macosko, "Graphene/polyurethane nanocomposites for improved gas barrier and electrical conductivity," Chemistry of Materials, vol. 22, no. 11, pp. 3441-3450, 2010.

[4] O. C. Compton, S. Kim, C. Pierre, J. M. Torkelson, and S. T. Nguyen, "Crumpled graphene nanosheets as highly effective barrier property enhancers," Advanced Materials, vol. 22, no. 42, pp. 4759-4763, 2010.

[5] H. Kim, A. A. Abdala, and C. W. Macosko, "Graphene/polymer nanocomposites," Macromolecules, vol. 43, no. 16, pp. 65156530, 2010.

[6] H. Yamaguchi, J. Granstrom, W. Nie et al., "Reduced graphene oxide thin films as ultrabarriers for organic electronics," Advanced Energy Materials, vol. 4, no. 4, Article ID 1300986, 2014.

[7] J. S. Lewis and M. S. Weaver, "Thin-film permeation-barrier technology for flexible organic light-emitting devices," IEEE Journal of Selected Topics in Quantum Electronics, vol. 10, no. 1, pp. 45-57, 2004.

[8] X. Li, W. Cai, J. An et al., "Large-area synthesis of high-quality and uniform graphene films on copper foils," Science, vol. 324, no. 5932, pp. 1312-1314, 2009.

[9] N. Kim, W. J. Potscavage, B. Domercq, B. Kippelen, and S. Graham, "A hybrid encapsulation method for organic electronics," Applied Physics Letters, vol. 94, no. 16, Article ID 163308, 2009.

[10] P. Mandlik, J. Gartside, L. Han et al., "A single-layer permeation barrier for organic light-emitting displays," Applied Physics Letters, vol. 92, no. 10, Article ID 103309, 2008.

[11] J. Meyer, P. Görrn, F. Bertram et al., "Al2O3/ZrO2 Nanolaminates as ultrahigh gas-diffusion barriersa strategy for reliable encapsulation of organic electronics," Advanced Materials, vol. 21, no. 18, pp. 1845-1849, 2009.

[12] G. Dennler, C. Lungenschmied, H. Neugebauer et al., "A new encapsulation solution for flexible organic solar cells," Thin Solid Films, vol. 511-512, pp. 349-353, 2006.
[13] J. Meyer, H. Schmidt, W. Kowalsky, T. Riedl, and A. Kahn, “The origin of low water vapor transmission rates through $\mathrm{Al} 2 \mathrm{O} 3 /$ $\mathrm{ZrO} 2$ nanolaminate gas-diffusion barriers grown by atomic layer deposition," Applied Physics Letters, vol. 96, no. 24, Article ID 243308,2010

[14] L. Moro, N. M. Rutherford, R. J. Visser et al., "Barix multilayer barrier technology for organic solar cells," in Proceedings of the Organic Photovoltaics VII, August 2006.

[15] J. H. Choi, Y. M. Kim, Y. W. Park et al., "Evaluation of gas permeation barrier properties using electrical measurements of calcium degradation," Review of Scientific Instruments, vol. 78, no. 6, Article ID 064701, 2007.

[16] H.-K. Seo, M.-H. Park, Y.-H. Kim, S.-J. Kwon, S.-H. Jeong, and T.-W. Lee, "Laminated Graphene Films for Flexible Transparent Thin Film Encapsulation," ACS Applied Materials \& Interfaces, vol. 8, no. 23, pp. 14725-14731, 2016.

[17] H. Klumbies, M. Karl, M. Hermenau et al., "Water ingress into and climate dependent lifetime of organic photovoltaic cells investigated by calcium corrosion tests," Solar Energy Materials \& Solar Cells, vol. 120, pp. 685-690, 2014.

[18] H. Klumbies, P. Schmidt, M. Hähnel et al., “Thickness dependent barrier performance of permeation barriers made from atomic layer deposited alumina for organic devices," Organic Electronics, vol. 17, pp. 138-143, 2015.

[19] Y. Su, V. G. Kravets, S. L. Wong, J. Waters, A. K. Geim, and R. R. Nair, "Impermeable barrier films and protective coatings based on reduced graphene oxide," Nature Communications, vol. 5, article 4843, 2014.

[20] T. Kim, J. H. Kang, S. J. Yang, S. J. Sung, Y. S. Kim, and C. R. Park, "Facile preparation of reduced graphene oxide-based gas barrier films for organic photovoltaic devices," Journal of Energy \& Environmental Science, vol. 7, no. 10, pp. 3403-3411, 2014.

[21] K. Choi, S. Nam, Y. Lee et al., "Reduced Water Vapor Transmission Rate of Graphene Gas Barrier Films for Flexible Organic Field-Effect Transistors," ACS Nano, vol. 9, no. 6, pp. 5818-5824, 2015.

[22] K.-H. Lee, J. Hong, S. J. Kwak, M. Park, and J. G. Son, "Spin self-assembly of highly ordered multilayers of graphene-oxide sheets for improving oxygen barrier performance of polyolefin films," Carbon, vol. 83, pp. 40-47, 2015.

[23] A. Ranade, N. A. D’Souza, R. M. Wallace, and B. E. Gnade, "High sensitivity gas permeability measurement system for thin plastic films," Review of Scientific Instruments, vol. 76, no. 1, Article ID 013902, pp. 1-13902, 2005.

[24] M. Surin, P. Leclére, R. Lazzaroni et al., "Relationship between the microscopic morphology and the charge transport properties in poly(S-hexylthiophene) field-effect transistors," Journal of Applied Physics, vol. 100, no. 3, Article ID 033712, 2006.

[25] N. S. Sariciftci, L. Smilowitz, A. J. Heeger, and F. Wudl, "Photoinduced electron transfer from a conducting polymer to buckminsterfullerene," Science, vol. 258, no. 5087, pp. 1474-1476, 1992.

[26] M. Manceau, A. Rivaton, J.-L. Gardette, S. Guillerez, and N. Lemaitre, "The mechanism of photo- and thermooxidation of poly(3-hexylthiophene) (P3HT) reconsidered," Polymer Degradation and Stability, vol. 94, no. 6, pp. 898-907, 2009.

[27] M. Manceau, S. Chambon, A. Rivaton, J.-L. Gardette, S. Guillerez, and N. Lematre, "Effects of long-term UVvisible light irradiation in the absence of oxygen on P3HT and P3HT: PCBM blend," Solar Energy Materials \& Solar Cells, vol. 94, no. 10, pp. 1572-1577, 2010. 
[28] H. Sojoudi and S. Graham, "Transfer-free selective area synthesis of graphene using solid-state self-segregation of carbon in Cu/Ni Bilayers," ECS Journal of Solid State Science and Technology, vol. 2, no. 6, pp. M17-M21, 2013.

[29] D. L. Matz, H. Sojoudi, S. Graham, and J. E. Pemberton, "Signature vibrational bands for defects in CVD single-layer graphene by surface-enhanced Raman spectroscopy," The Journal of Physical Chemistry Letters, vol. 6, no. 6, pp. 964-969, 2015.

[30] M. S. A. Abdou, F. P. Orfino, Y. Son, and S. Holdcroft, "Interaction of oxygen with conjugated polymers: Charge transfer complex formation with poly(3-alkylthiophenes)," Journal of the American Chemical Society, vol. 119, no. 19, pp. 4518-4524, 1997.

[31] H. Sojoudi, J. Baltazar, L. M. Tolbert, C. L. Henderson, and S. Graham, "Creating graphene p-n junctions using self-assembled monolayers," ACS Applied Materials \& Interfaces, vol. 4, no. 9, pp. 4781-4786, 2012.

[32] J. Baltazar, H. Sojoudi, S. A. Paniagua et al., "Facile formation of graphene P-N junctions using self-assembled monolayers," The Journal of Physical Chemistry C, vol. 116, no. 36, pp. 19095-19103, 2012.

[33] J. Baltazar, H. Sojoudi, S. A. Paniagua et al., "Photochemical doping and tuning of the work function and dirac point in graphene using photoacid and photobase generators," Advanced Functional Materials, vol. 24, no. 32, pp. 5147-5156, 2014.

[34] J. Granstrom, J. S. Swensen, J. S. Moon, G. Rowell, J. Yuen, and A. J. Heeger, "Encapsulation of organic light-emitting devices using a perfluorinated polymer," Applied Physics Letters, vol. 93, no. 19, Article ID 193304, 2008.

[35] A. J. Ferguson, J. L. Blackburn, J. M. Holt et al., "Photoinduced energy and charge transfer in P3HT:SWNT composites," The Journal of Physical Chemistry Letters, vol. 1, no. 15, pp. 24062411, 2010.

[36] W. Zhao, K. Shi, and Z. Lu, "Greatly enhanced ultrabroadband light absorption by monolayer graphene," Optics Expresss, vol. 38, no. 21, pp. 4342-4345, 2013.

[37] G. Pirruccio, L. Martín Moreno, G. Lozano, and J. Gómez Rivas, "Coherent and broadband enhanced optical absorption in graphene," ACS Nano, vol. 7, no. 6, pp. 4810-4817, 2013.

[38] H. Sojoudi, J. Baltazar, C. Henderson, and S. Graham, "Impact of post-growth thermal annealing and environmental exposure on the unintentional doping of CVD graphene films," Journal of Vacuum Science \& Technology B, Nanotechnology and Microelectronics: Materials, Processing, Measurement, and Phenomena, vol. 30, no. 4, p. 041213, 2012.

[39] V. Shrotriya, J. Ouyang, R. J. Tseng, G. Li, and Y. Yang, "Absorption spectra modification in poly(3-hexylthiophene): methanofullerene blend thin films," Chemical Physics Letters, vol. 411, no. 1-3, pp. 138-143, 2005.

[40] M.-C. Wu, H.-C. Liao, H.-H. Lo et al., "Nanostructured polymer blends (P3HT/PMMA): Inorganic titania hybrid photovoltaic devices," Solar Energy Materials \& Solar Cells, vol. 93, no. 6-7, pp. 961-965, 2009.

[41] M.-C. Wu, H.-C. Liao, Y. Chou et al., "Manipulation of nanoscale phase separation and optical properties of P3HT/PMMA polymer blends for photoluminescent electron beam resist," The Journal of Physical Chemistry B, vol. 114, no. 32, pp. 10277-10284, 2010.

[42] K. Tremel and S. Ludwigs, "Morphology of P3HT in thin films in relation to optical and electrical properties," Advances in Polymer Science, vol. 265, pp. 39-82, 2014.
[43] F. J. Nelson, V. K. Kamineni, T. Zhang, E. S. Comfort, J. U. Lee, and A. C. Diebold, "Optical properties of large-area polycrystalline chemical vapor deposited graphene by spectroscopic ellipsometry," Applied Physics Letters, vol. 97, no. 25, Article ID 253110, 2010. 


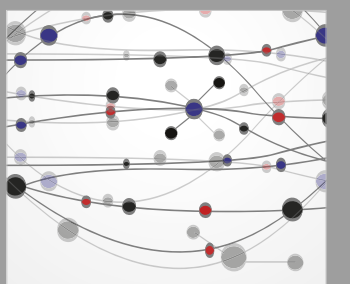

The Scientific World Journal
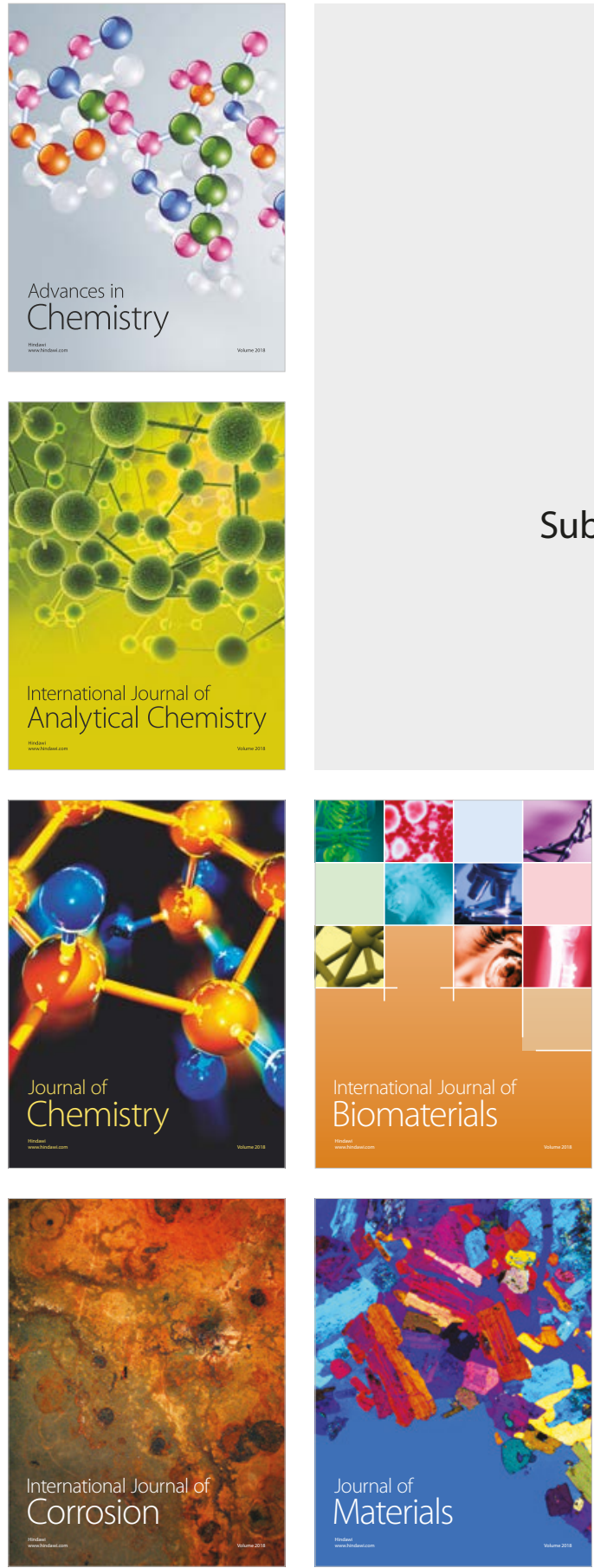

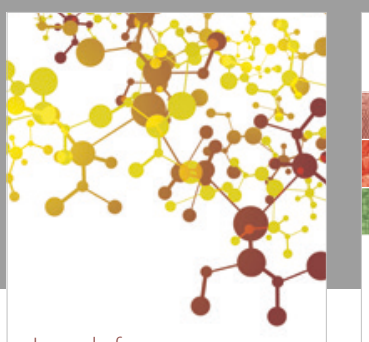

Journal of

Applied Chemistry
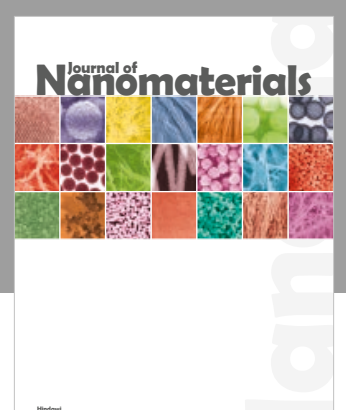

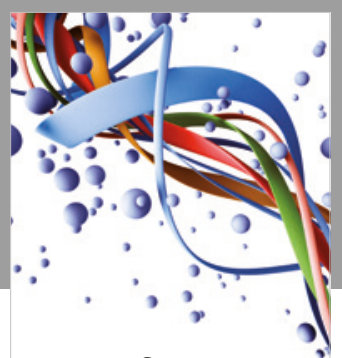

Scientifica

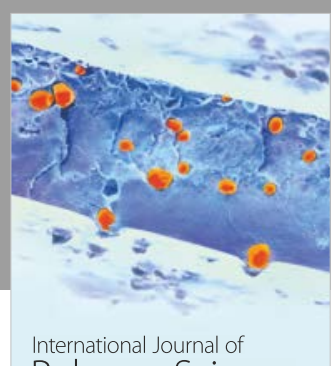

Polymer Science

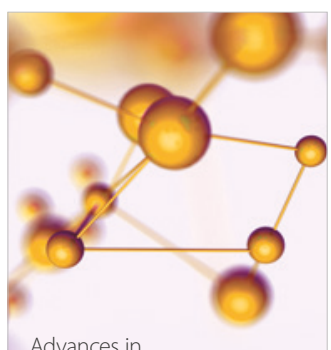

Physical Chemistry
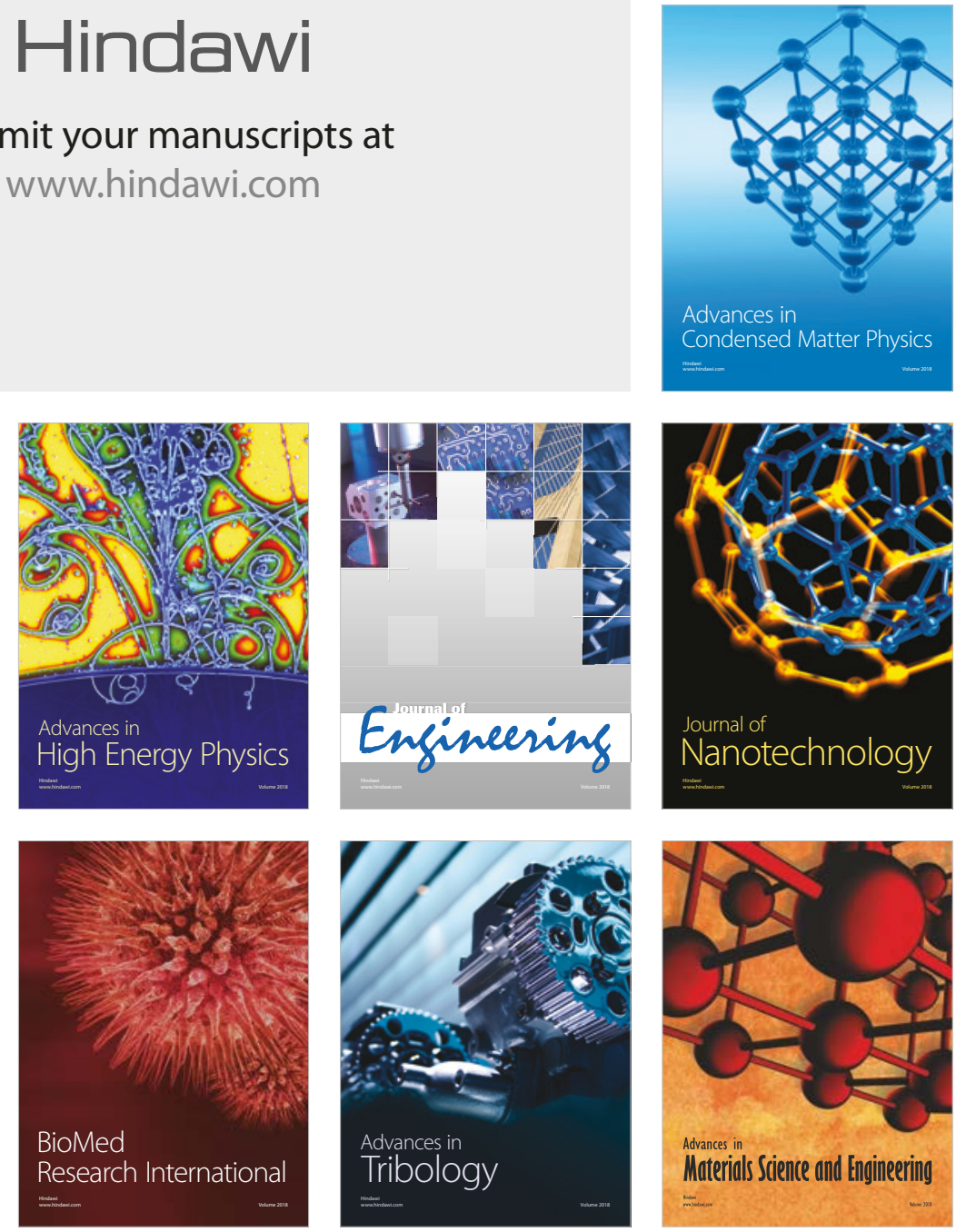